\title{
Multiple Organ Dysfunction Syndrome In Major Burns Patients
}

\author{
Alam MS1, Begum $\mathrm{SH}^{2}$.
}

\begin{abstract}
Organ dysfunctions are frequent after major burn trauma. Development of MODS in severely burned patients is highly fatal. The present study was conducted to elucidate the etiology and quantify the Multiple Organ Dysfunction (MODS) in major burn patients. "Multiple organ dysfunctions in major burnt patients" was a prospective analytic study.

A total of thirty patients were included in the study, which was conducted in the Burn and Plastic Surgery unit, Bir Hospital, Kathmondu, Nepal over the period of eighteen months from January 2002 to June 2003. Patients with acute major burn i.e. 24 hours from the time of injury were admitted and included in the study. All patients were initially treated in the ICU of burn unit. Among 30 patients, 25 patients were in the age group 15-30 years and 5 patients were in the 31-60 years age group. 12 patients were male and 18 were female. $93.3 \%$ of patients in this study had sustained flame burn and the chief mode of burn injury was accidental $50 \%$ whereas $46.7 \%$ were suicidal. $66.7 \%$ of the patients had 20\% - 50\%TBSA and 33.3\% had sustained 50\% - 90\% TBSA burn. Majority of the patients were under went early excision and SSG with regular dressing. $63.3 \%$ (19 patients) develop multiple organ dysfunctions and most frequently involved organ was kidneys and followed by lungs, liver and pancreas. Among the multiple organ failure patients, 10 had developed septicemia. None of the patients were found to have developed cardiac failure. The mortality in the study population was $46.7 \%$, all of them had MODS and 9 of them had inhalation injury. It was also seen that MODS can

1. Dr. Mohammad Shaha Alam, MBBS, MS (Surgery), FICS (USA) Surgery Specialist

District Sadar Hospital \& Cox's Bazar Medical College,

Cox's Bazar, Bangladesh.

2. Dr. Syeda Humaira Begum, MBBS, MS

Fouad Al Khateed Hospital, Cox’s Bazar
\end{abstract}

\section{Introduction:}

The burn injury can be one of the most serious and devastating form of trauma that man can sustain. Millions of people around the world are hospitalized for the treatment of burns each year and thousands die. A burn by definition is a tissue injury from thermal application (both Heat and Cold), chemical contact, electrical injuries and radiation ${ }^{1}$.

Burn is not only a superficial and localized injury affecting only the skin; it is systemic, and involves all systems of the body. This requires specialized treatment by a team of medical and nursing personnel. The aim of this burn team is to prevent the patient's death, heal the wound and then to rehabilitee them in the society. The appropriate management of burns requires knowledge, skill and a dedicated team. Positive out comes are enhanced through immediate intervention by first responders in the emergency department. Advances in treatment are based on improved understanding of resuscitation, enhanced wound coverage, better support of the hyper metabolic response to injury, more appropriate infection control and improved treatment of inhalation injuries ${ }^{2}$. The quality of burn care is reflected not only by surgery but also by the long-term function and appearance $^{3}$.

The first introduction of burn centers in $1945^{4}$ heralded a rapid improvement in survival and reduction of morbidity of burn patients. This provided the basis for regional specialty treatment centers in other disciplines. Before introduction of modern burn centers survival from a burn injury involving $30 \%$ of the total body surface area (TBSA) at any age was unpredictable.

Today, children survive burns affecting more than $90 \%$ of TBSA; young and middle-aged adults with more than $70 \%$ burns survive routinely in developed countries 5 .

In our context, most common cause of burn is accidental flame burn related to kerosene stove explosion and an increasing suicidal death by burning. In Nepal, due to mountainous terrain transportation and financial constraints, the victims are not able to receive prompt and proper medical care. Many of these patients die without any primary care. Those who survive, come to us very late 
without proper resuscitation and with complications like infected wounds, chronic ulcers, and contractures.

Bir Hospital is a tertiary level hospital in Nepal which has developed a modern burn unit with the aim of improving survival of burnt patients in a multidisciplinary team approach. This setup is one of its kinds in Nepal.

Recent advances in the knowledge of pathophysiology of burn's shock and fluid replacement therapy and the current practice of early wound excision and closure have reduced death in severely burned patients. In spite of a remarkable decrease in fatality rates over the past few decades, burn trauma continues to be a leading cause of morbidity and mortality ${ }^{6}$.

Multiple Organ dysfunction syndrome (MODS) are frequent after major burn trauma ${ }^{7}$. Most of the patients who die do so as a consequence of MODS and that, in spite of having had treatment for several infectious episodes, they still in the long run die of uncontrolled infection. The infections that incited the inflammatory process may have been generally controlled, but some resultant systemic inflammation might have persisted. This hypothesis is consistent with both the 'multi - hit' hypothesis of Frye ${ }^{8}$ and the concept of the systemic inflammatory response syndrome ${ }^{9}$.

MODS is felt to be caused by several possible 'engines', 10 including infection, tissue injury, bacterial translocation from the gut, ${ }^{11}$ and inadequate oxygen delivery ${ }^{12}$.

MODS in this setting are a consequence of uncontrolled systemic inflammation that occurs initially in response to soft tissue injury or infection. The routine investigation often fails to predict the development of MODS. It is imperative to include certain laboratory investigation to detect the development of MODS so that remedial measures can be taken at an early stage, thereby, decreasing the morbidity and mortality. The concept of MODS leading to death in burned patients is relatively new. High mortality resulting from MODS in burn patients was first reported in $1973^{13}$.

The prevention of MODS is likely to be more effective in burn patients if proper modern scientific treatment is started from the time of the patient's entry in the emergency. Once admitted early wound excision and closure is done to minimize sepsis and inflammation, support of the gut through enteral nutrition and general haemodynamic support to ensure adequate oxygen perfusion of the gut and peripheral tissues is started ${ }^{14}$.

Aim of the study was to elucidate the etiology and incidence of multiple organ failure in major burn patients and to determine the number and percentage of patients develop MODS in major burns as well as to find out the probable causes of MODS in major burnt patients.

\section{Methodology}

This was carried out as a hospital based prospective observational study.

The study was conducted in the Burn and Plastic surgery unit, with the support of department of Pathology, Biochemistry, Cardiology and Cardiothoracic and Vascular surgery unit Bir Hospital.

The study was conducted over a period of one year and six months from January 2002 to June 2003. Patients with acute major burns( within 24 hours) attending the Bir Hospital emergency within 24 hours of injury and who got admitted in the burn unit were taken in to consideration for selection as study subjects. A total of 30 cases, major burn of any nature (flame, electrical, chemical etc.) have been included in the study.

The patients with acute burn injuries who had sustained $>30 \%$ of total body surface area of burns were admitted in the acute burn unit were studied. Information collected included age, sex, date of burn, type of burn, presence or absence of inhalation injury and mode of injury. Inhalation injury was considered present if the fire had occurred in a closed space.

The severity of the burn was estimated by percentage of total body surface area (TBSA \%) using "rule of nine" and wound depth was categorized as degree of burn. Study included all patients who died, including those who died within 72 hours of admission. The age of the patients varied from 15 to 60 years. All the patients were admitted in the burn ICU and were resuscitated. The general wound care for the patients with major burns in the ICU was daily dressing changes with silver sulfadiazine. And early wound excision biological closure with split skin graft (SSG) being effected within the first post injury week and continued in sequential manner until the wound was completely covered. Nutrition was started via the enteral route usually after 24 hours of burn. Parenteral nutrition was used only for those who were unable to tolerate the enteral route.

Organ failure was assessed by the criteria used by Aikawa 14 as shown in Table -1 .

The diagnosis of septicemia was made using clinical signs of infection and isolation of pathogens by blood culture. Any patient who had involvement of two or more organ failure was labeled as patient of Multiple Organ Dysfunction Syndrome.

The investigations were done immediately after admission then after 48 hours and whenever it was necessary. 
Table I. Criteria used for MODS

\begin{tabular}{|l|l|}
\hline Lungs & Arterial blood gas analysis, \\
& $\mathrm{PH}<7.30$, \\
& $\mathrm{PaO} 2<60 \mathrm{~mm} \mathrm{Hg}$ \\
\hline Kidneys & Blood Urea $>60 \mathrm{mg} / \mathrm{dl}$ \\
& Serum Creatinine $>2.0 \mathrm{mg} / \mathrm{dl}$ \\
\hline Liver & SGOT $>30 \mathrm{IU} / \mathrm{L}$ \\
& SGPT $>30 \mathrm{IU} / \mathrm{L}$ \\
& Alk. Phosphatase $>10 \mathrm{KAU} / \mathrm{L}$ \\
& S. bilirubin $>2.0 \mathrm{mg} / \mathrm{dl}$ \\
\hline Heart (Myocardium Enzyme Spectrum) & CPK-MB $>2.0 \mathrm{IU} / \mathrm{L}$ \\
& SGOT $>80 \mathrm{IU} / \mathrm{L}$ \\
& LDH $>300 \mathrm{~L} / \mathrm{L}$ \\
\hline Pancreas & Serum Amylase $>300 \mathrm{SU} / \mathrm{L}$ \\
& Serum Lipase $>300 \mathrm{IU} / \mathrm{L}$ \\
\hline
\end{tabular}

Informed consent from patient or patient party was taken before included in the study. All data were reviewed in details and maintained prospectively. FDP are only obtained when there was a clinical suspicion of DIC. Daily MODS score determinations were made for pulmonary, renal, hepatic, pancreas and cardiovascular system, according to the published threshold values.

In spite of all follow up, blood culture report plus information was also recorded regarding sepsis according to American College of Chest Physicians/Society of Critical Care Medicine Consensus Conference: a definition for sepsis or Diagnosis requires that two or more of the following criteria be met.

1. Temperature $>388 \mathrm{C}$ or $<368 \mathrm{C}$

2. Heart rate $>90 \mathrm{bpm}$

3. Respiratory rate $>20$ breaths $/ \mathrm{min}$ or $\mathrm{PaCO} 2<32 \mathrm{mmHg}$

4. $\mathrm{WBC}>12,000 / \mathrm{mm} 3$ or $<4000 \mathrm{~mm} 3$ or $10 \%$ immature (band) forms

Analysis of data was performed using SPSS 10.0 statistical software. Values were reported as mean \pm SD. Chi-Square test was used for statistical values.

\section{Result}

Out of 30 patients with the major burns, the commonest age group was 15 - 30 years with 25 patients $(83.3 \%)$ and 31 60 years with 5 patients $(16.7 \%)$.There were 18 females $(60 \%)$ and 12 males $(40 \%)$, the female and male ratio was 1.5: 1 .

Out of 30 patients 10 of them came from out side of Kathmandu. And 20 patients from Kathmandu valley. The majority of the patients in the present study sustained injury from flame burn $93.3 \%$ while 2 patients $(6.7 \%)$ had sustained electrical burn.

The mode of burn injury was chiefly accidental, 15 patients (50\%). The second most common mode of burn was suicidal which were 14 patients and 1 patient had sustained
Majority (66.7\%) of the patients had 20\%- 50\% TBSA burns while 10 patients $(33.3 \%)$ had $50 \%-90 \%$ TBSA burns. When only 2nd and 3rd degree burns were taken in to consideration according to the mentioned protocol.

Nine patients with major burn sustained inhalation injury and rest of 21 patients had found no sign of inhalation injury. Out of 30 patients of major burns 10 patients developed post burn septicemia among them 5 patients had associated shock during early treatment. And 20 patients had found no signs of septicemia except who died due to extensive burn before diagnosis of septicemia were established. [Fig:1]

In our study multiple organ dysfunctions developed in 19 patients where MODS defined as "two or more organ dysfunction". Six patients had developed 4 organs, four patients had 3 organ and nine patients had 2 organ dysfunction. Single organ dysfunction developed in 4 patients while no organ dysfunction has been seen in 7 patients. Lungs and kidney were found to be the most commonly affected organ in 30 patients, kidneys in 22 patients and lungs in11patients. No cardiac dysfunction was found in the study population. Of the four patients who developed single organ failure, all of them had developed only renal failure. Of the 10 patients who developed septicemia as revealed by positive blood cultures were developed MODS and died. All the 9 patients sustained inhalation injury were developed lungs failure followed by MODS and subsequently died. UTI was found more common in the renal failure patients ${ }^{12}$.

In the study 14 patients were dead due to major burns; all of them developed MODS except one who developed single organ (Kidneys) failure. So Multiple Organ Dysfunction developed $63.3 \%$ and over all mortality rates in this study was $46.7 \%$. [Fig: 2]

Fig: 1 - num. pt. develops septicaemia

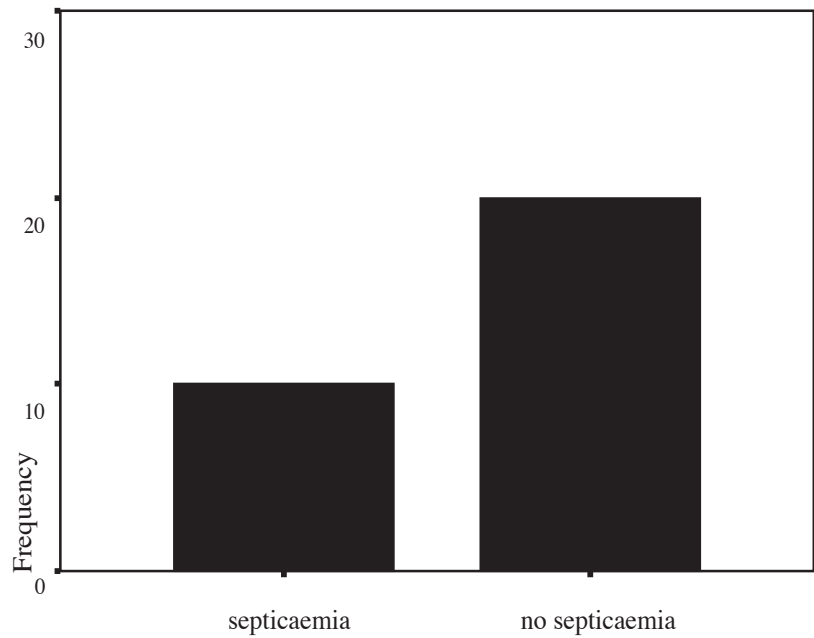




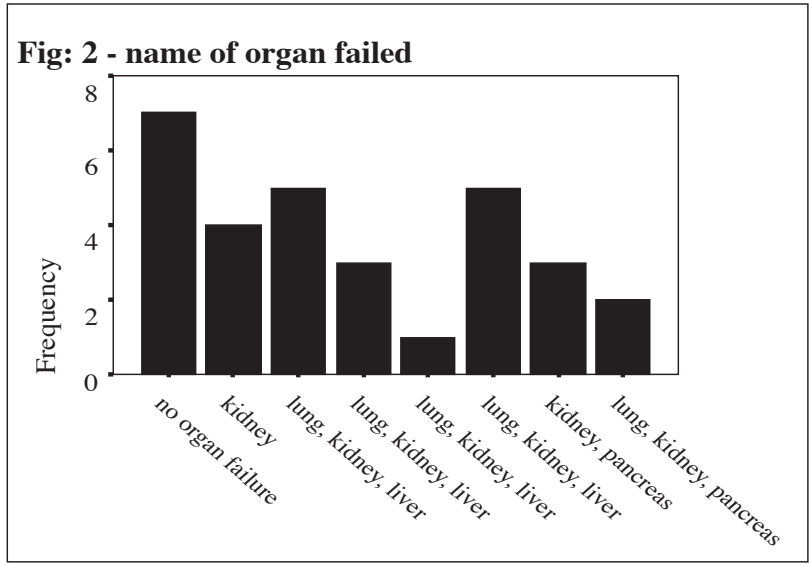

\section{Discussion}

The concept of multiple Organ Dysfunction leading to death in major burn patients is relatively new. A more complete description of organ dysfunction or failure in patients with burn trauma is a fairly complex. The uncontrolled systemic inflammation, triggered by multiple small infections, leads to multiple organ failure and death is a commonly held belief. The study of Marshall ${ }^{15}$ and colleagues was one of the first studies to evaluate multiple organ dysfunctions after burn trauma. They studied 168 patients with burns over $40 \%$ TBSA and objectively defined cardiovascular, pulmonary, and renal failure; they considered failure as an all-or-none phenomenon rather than as a continuum of increasing severity. Multiple organ failure carried a $98 \%$ mortality in the study by Marshall, compared with $63.3 \%$ in our study. The high mortality resulting from a combination of vital organ failure was first reported by Tilney et 12 and two years later Baue ${ }^{13}$ reported the clinical significance of multiple progressive sequential systemic failure seen in variety of surgical patients. Aikawa ${ }^{14}$ advocated a new entity of clinical syndrome of Multiple Organ Failure in burned patients and showed that 26 of the 54 patients with severe burns had Multiple Organ Failure.

The incidence of MODS in our study is $63.3 \%$. It is also significant that patients with inhalation injury or septicaemia had absolute incidence of MODS. Of the 30 patients associated with shock was 9 patients, who developed organ failure early and all of them found kindney dysfunction. Association of multiple organ failure was found significantly higher.

Ten patients who developed septicemia had a higher association with multiple organ dysfunctions. Evidence of this incidence was also reported by Fry ${ }^{8}$. We have also found 3 patients who developed multiple organ failure without any strong evidence of infection but they had sustained respiratory injury or burn shock. Aikawa ${ }^{15}$ also encountered similar findings and had attributed these to endotoxaemia possible originating from patient's intestinal flora, which may be result of an impaired intestinal barrier has also been documented by Bounous ${ }^{16}$ and Deitsch ${ }^{17}$ where normal intestinal barrier function is affected by hypovolaemic shock. Marshall ${ }^{15}$ also described similar etiology in MODS.

In addition to quantifying organ dysfunction, we estimated the incidence of sepsis and its most severe expression after burn trauma. Over one third patients in the cohort developed sepsis according to clinical criteria and presence of positive blood culture. The risk factors for severe sepsis/septic shock were similar to the risk factors for severe multiple organ dysfunction.

Our study is limited in its sample size as shown by the small absolute numbers of patients with sepsis, shock, or MOF. Furthermore, the small number of patients with inhalation injury limits our ability to evaluate this potentially important risk factor for organ dysfunction. Few of our patients died due to extensive burn with in few hours after admission.

In the present clinical study, it has been shown that the occurrence of MODS during early stage post burn was mainly associated with cases of respiratory dysfunction, severe shock and/or systemic sepsis. Moreover, a severe major burn was shown to be potent instigators of MODS, because patients under these conditions are very vulnerable to severe shock and sepsis. In order to verify the importance of these four etiological factors (severity of burns, inhalation injury, shock and sepsis) in the development of MODS.

In some instances, MODS may develop directly after shock and in some others severe shock was not apparent, systemic sepsis emerges early and is followed by MODS. In the later instance, etiological factors can be many, severe inhalation injury often plays the major role. In both severe shock and severe inhalation injury, prolonged hypoxia is the common pathophysiological basis or insult that induces systemic sepsis and/or MODS. In this series of studies it was found that severe burn shock often occurred in those severe burn cases to which early or/and proper resuscitation was not delivered. Therefore, the period of low blood perfusion and hypoxia were apparently prolonged. Consequently, severe ischemic tissue damage was inevitably resulted. Also, as soon as fluid replacement was started, so-called 'reperfusion injury' would be superimposed on the ischemic tissue damage, which was widespread and may occur in any tissue or organ, involving the endothelial cells which were especially vulnerable to hypoxia. The findings of other study also demonstrated an increase of circulatory platelet aggregates and microthrombosis in visceral microvasculatures. These changes might result in microcirculatory disturbance and ischemic-hypoxic damage to tissues

This observation might be attributed to the release of tissue products following reperfusion of ischemic tissues, suggesting that more severe tissue ischemia and reperfusion damage had occurred. It has been well recognized that oxygen free radicals are the major causes of reperfusion damage. 
We had lot of limitations to do frequent biochemical test. Our evaluation of potential risk factors is not complete, but it was our intention to first provide objective estimates of the incidence and consequence of multiple organ dysfunctions in patients with major burn trauma. Our study provides an important first step to subsequent detailed study of important risk factors for organ failure in Nepal. For example, other investigators have found indicators of shock, tissue injury, and existing patient factors to be important determinants of MODS after trauma. A similar comprehensive analysis of risk factors for organ dysfunction and failure after burn trauma will be an important next step.

In summary, there are several reports an objective method for scoring organ dysfunction after burn trauma that is derived from readily available clinical and laboratory data that allows the course of organ dysfunction to be followed over the post injury period, and that promotes comparison to other studies which was not followed in the present study. Multiple organ dysfunction and severe sepsis/septic shock are both related to burn size, age, and inhalation injury which can be partially overcome by a scientific and proper team approach to a burn victim. It is now well documented that MODS is a frequent and serious complication in major burned patients leading to a high mortality rate. The control of septicemia, adequate management of shock and support to the respiratory system should reduce the mortality of burns due to Multiple Organ Dysfunction.

The introduction of new terminology, such as MODS and SIRS represents only a small step toward the development of a descriptive framework that will allow the clinician to provide optimal care for the critically ill burnt patient. The unanswered challenges are many: early and definitive diagnosis of infection, the selection of optimal clinical or biochemical parameters to characterize the systemic inflammatory response and the development of consensus criteria for organ dysfunction that can be used as for diagnosis and favorable outcome.

\section{Reference}

1. Russel RCG, Williums NS, Bulstrode CJK. Bailey \& Love's Short Practice of Surgery. 23rd ed. Aronld: 2000;188-189.

2. Martyn JAJ. Acute management of the burn patient:WB.SaundersCompany;1990
3. Sheridan R.L. Death in burn unit : sterile multiple organ failure. Burns. 1998;24:307-311

4. Glenn D, Warden, David M. Principal of Surgery Schwartz. 7thed. Heimbach 1999;223-224.

5. Sheridan RL, Ronald G. Oxford Surgery. 2nd ed. Tompkins: 1995;3585-3589

6. Ryan C M . Objective estimates of the probability of death from burn injury. N Engl J Med. 1998;338: 362-366

7. Cumming J. Objective estimates of the incidence and consequences of multiple organ dysfunction and sepsis after burn trauma

8. Fry DE, Pearlstein MD. Multiple system organ failure. The role of uncontrolled infection. Archive of Surgery. 1980;115:136-140

9. Bone R.C, Fisher. Sepsis syndrome; A valid clinical entity, Methylprednisolone, severe sepsis study group, clinical care medicine.1989;17:389-393

10. Goodwin C W. Multiorgan failure, clinical overview of syndrome, Journal of trauma.1990;30:163-165

11. Cain S M. Supply dependency of oxygen uptake in ARDS Myth \& reality, American J of Medical science.1984;288:119-124

12. Tinly N L, Baily G L, Morgan A P. Sequential system failure after rupture of ABD, Aortic aneurysm,an unsolved problem in post operative care. Ann surgery; 1973;178:117

13. Baue A E; What's in a name?An acronym or a response? Americanjournal of Surgery; 1993;165:299-301

14. Aikawa, Shinozawa Y, Ishibika K. Clinical analysis of multiple organ failure in burned patients. Burns .1987; 13:103-109

15. Marshall WG. Dimick AR. The natural history of major burn with multiple system failure.

J. Trauma. 1983;23:102-105

16. Bounous, G.: Acute necrosis of the intestinal mucosa. Gastroenterology 82;1457:1982

17. Deitch E.A., Sittig K., Li M., Berg R., Specian, R.D. Obstructive jaundice promotes bacterial translocation from the gut. Am. J. Surg.1990;159:79 